\title{
A note on the ranks of set-inclusion matrices
}

\author{
D. de Caen \\ Department of Mathematics and Statistics \\ Queen's University \\ Kingston, Ontario, Canada K7L 3N6 \\ decaen@mast . queensu.ca
}

Submitted: June 11, 2001; Accepted: June 16, 2001

\begin{abstract}
A recurrence relation is derived for the rank (over most fields) of the set-inclusion matrices on a finite ground set.
\end{abstract}

Given a finite set $X$ of say $v$ elements, let $W=W_{t, k}(v)$ be the (0,1)-matrix of inclusions for $t$-subsets versus $k$-subsets of $X: W_{T, K}=1$ if $T$ is contained in $K$, and 0 otherwise. These matrices play a significant part in several combinatorial investigations, see e.g. ([2], Thm. 2.4).

Let $F$ be any field, and let $r_{F}(M)$ denote the rank of $M$ over $F$.

Theorem. If $(k-t) \neq 0$ in the field $F$, then

$$
r_{F}\left(W_{t, k}(v+1)\right)=r_{F}\left(W_{t, k-1}(v)\right)+r_{F}\left((k-t+1) W_{t-1, k}(v)\right) .
$$

Proof. The block-matrix identity

$$
\left[\begin{array}{cc}
I & -A \\
0 & I
\end{array}\right]\left[\begin{array}{cc}
A B & 0 \\
B & B C
\end{array}\right]\left[\begin{array}{cc}
I & -C \\
0 & I
\end{array}\right]=\left[\begin{array}{cc}
0 & -A B C \\
B & 0
\end{array}\right]
$$

implies that, over any field $F$,

$$
r_{F}\left(\left[\begin{array}{cc}
A B & 0 \\
B & B C
\end{array}\right]\right)=r_{F}(B)+r_{F}(A B C) .
$$

The set-inclusion matrix has the block-triangular decomposition

$$
W_{t, k}(v+1)=\left[\begin{array}{cc}
W_{t-1, k-1}(v) & 0 \\
W_{t, k-1}(v) & W_{t, k}(v)
\end{array}\right],
$$


as may be seen by fixing $x$ in $X$ and classifying $t$-sets and $k$-sets according to whether $x$ belongs to them or not. Further, there is the elementary product formula

$$
W_{t, k}(v) W_{k, l}(v)=\left(\begin{array}{c}
l-t \\
k-t
\end{array}\right) W_{t, l}(v)
$$

whose proof is left as a straightforward exercise. Using (4), one may re-write (3) as

$$
W_{t k}(v+1)=\left[\begin{array}{cc}
\frac{1}{(k-t)} W_{t-1, t}(v) W_{t, k-1}(v) & 0 \\
W_{t, k-1}(v) & W_{t, k-1}(v) W_{k-1, k}(v) \frac{1}{(k-t)}
\end{array}\right]
$$

and so (2) is applicable:

$$
\begin{aligned}
r_{F}\left(W_{t, k}(v+1)\right) & =r_{F}\left(W_{t, k-1}(v)\right)+r_{F}\left(W_{t-1, t}(v) W_{t, k-1}(v) W_{k-1, k}(v)\right) \\
& =r_{F}\left(W_{t, k-1}(v)\right)+r_{F}\left((k-t+1) W_{t-1, k}(v)\right),
\end{aligned}
$$

which completes the proof of (1).

Corollary Over the rational field $Q, r_{Q}\left(W_{t, k}(v)\right)=\left(\begin{array}{c}v \\ t\end{array}\right)$, provided $k+t \leq v$.

Proof. This is very easy using (1): note that the condition " $k+t \leq v$ " is inherited by the triples $(t, k-1, v-1)$ and $(t-1, k, v-1)$; so the result follows by induction.

The corollary is a well known result, first proved by Gottlieb [3]. Wilson [4] has worked out the modular ranks of $W_{t, k}(v)$. Unfortunately, the condition $(k-t) \neq 0$ in the hypothesis of our theorem precludes a new proof of Wilson's theorem via our recursive formula. In the special case when the characteristic $p$ of $F$ is larger than $k$, our recursion does apply, with the same conclusion and proof as the above corollary.

In conclusion, we raise the question as to whether there is a $q$-analogue of formula (1), i.e., for the $(0,1)$-inclusion matrix $W_{t, k}^{(q)}(v)$ of $t$-dimensional subspaces versus $k$-dimensional subspaces of a $v$-dimensional space over $G F(q)$; see [1], where the $F$-rank of $W_{t, k}^{(q)}(v)$ is computed when $\operatorname{char}(F)$ does not divide $q$.

Acknowledgement Support has been provided by a grant from NSERC.

\section{References}

[1] A. Frumkin and A. Yakir, "Rank of inclusion matrices and modular representation theory", Israel J. Math. 71 (1990), 309-320.

[2] C. D. Godsil, "Tools from linear algebra", in Handbook of Combinatorics (eds., Graham, Grötschel, Lovász), MIT press 1995, pp. 1705-1748.

[3] D. H. Gottlieb, "A class of incidence matrices", Proc. Amer. Math. Soc. 17 (1966), 1233-1237.

[4] R. M. Wilson, "A diagonal form for the incidence matrix of $t$-subsets vs. $k$-subsets", European J. Combin. 11 (1990), 609-615. 